\title{
Fourier Transform Applied to Alternating Temperature Calculation
}

\author{
Françoise Depasse, Nathalie Trannoy and Philippe Grossel \\ Unité de Thermique et d'Analyse Physique, Laboratoire d'Énergétique et d'Optique, \\ Faculté des Sciences de Reims, BP 1039, 51687 Reims Cedex 2, France
}

(Received October 4; accepted October 18, 1996)

$\begin{aligned} & \text { PACS.02.60.-x } \text { - Numerical approximation and analysis } \\ & \text { PACS.02.70.-c } \text { - Computational techniques } \\ & \text { PACS.61.16.Ch }- \text { Scanning probe microscopy: scanning tunneling, atomic force, } \text { scanning optical, magnetic force, etc. } \\ & \text { PACS.44.10.+i }- \text { Heat conduction (models, phenomenological description) } \\ & \text { PACS.44.50.+f }- \text { Thermal properties of matter (phenomenology, experimental techniques) }\end{aligned}$

\begin{abstract}
The alternating temperature field variations in a conductive medium follow an equation similar to the wave equation. Thus, several mathematical tools known for a long time in optics can be used for heat diffusion calculations. Especially, the plane wave expansion appears very powerful. It is used in a nanothermic example where theoretical results are compared with experimental ones.
\end{abstract}

Résumé. - Les variations du champ de température alternative dans un milieu conductif sont régies par une équation du type équation d'ondes. C'est pourquoi plusieurs outils mathématiques connus depuis longtemps en Optique peuvent être utilisés pour des calculs de diffusion de la chaleur. En particulier, la décomposition en ondes planes apparait comme très efficace. Elle est utilisée dans un exemple de nanothermique, où les résultats du calcul sont comparés à ceux fournis par l'expérience.

\section{Introduction}

Numerous non-destructive evaluation experiments use thermophysical characterization of surface or sub-surface profiles. Their increasing accuracy makes possible, nowadays, the detection of submicrometric defects hidden in a solid sample. Thus, it is convenient to speak about "thermal microscopy" [1-4].

A large part of these thermal measurement methods use an harmonic modulated heat source which generates in the sample an alternating temperature field. In this paper, we show that the Fourier transform constitutes a precious tool for the conductive medium temperature study, especially in the harmonic case. Indeed, in spite of the physical nature differences between these phenomena, several mathematical methods used for a long time in acoustics and in optics can be extremely helpful in many alternating thermal diffusion problems. 
Among these common methods, let us mention the discrete Fourier transform (finite sample) [5,6] and the Hankel transform (cylindric symmetry problem) [7] which are not described in the present paper. We limit ourself here to the most fundamental tool: the continuous Fourier transform.

\section{The Temperature Field in a Solid Medium}

\subsection{Thermal Measurements}

Thermal measurement constitutes a very accurate method to obtain information about many physical phenomena, in a non destructive way. For example, the sample temperature depends on the thermal energy received by this sample. It is also a function of the sample shape (including inner defects, like fissures or inhomogeneities). The solid temperature depends also on chemical and physical (crystalline phase) composition of the sample.

Some measurement methods provide information about the sample general temperature, by detecting electric resistivity or refractive index global variations. When using such methods, one assumes evidently that the temperature is the same in the bulk sample. Other methods are more sophisticated and give access to the surface temperature map. Let us mention infrared detection and mirage effect [8]. Let us mention also all the scanning methods, which use a probe thermally coupled with the sample. In these cases, the probe temperature, which is globally known, depends on the sample region around the probe [4,9]. The surface temperature map obtained by measurement is used to reconstitute the temperature in the bulk of the sample, as a first step in solving the inverse problem. That is why this paper is concentrated on the calculus of the thermal field in a conductive medium.

\subsection{Heat Diffusion Equation and Boundary Conditions}

We study here the temperature variations in a solid sample, for times longer than the microsecond. Thus, we do not use the "hyperbolic equation" describing the non-diffusing behaviour of heat during very short times [10-12]. We assume also that temperature variations are small enough to leave the sample thermal coefficients unchanged. Under these assumptions, the temperature spatio-temporal variations in the sample follow the so-called "Fourier equation" [5]

$$
\rho(\mathbf{r}) C(\mathbf{r}) \frac{\partial T(t, \mathbf{r})}{\partial t}=\nabla[\lambda(\mathbf{r}) \nabla T(t, \mathbf{r})]+\sigma(t, \mathbf{r})
$$

with the following nomenclature: $\mathbf{r}$ the position vector, $t$ the time, $\rho$ the volumic mass, $C$ the thermal capacity density (for a constant volume), $T$ the temperature, $\lambda$ the thermal conductivity, and $\sigma$ the internal energy production rate per time and volume unit.

In the special case where the solid sample is homogeneous and isotropic, we may use the (constant) thermal diffusivity, defined as

$$
a=\frac{\lambda}{\rho C}
$$

and write equation (1) in a shorter way

$$
\Delta T(t, \mathbf{r})-\frac{1}{a} \frac{\partial T(t, \mathbf{r})}{\partial t}=-\frac{\sigma(t, \mathbf{r})}{\lambda}
$$

Two kinds of boundary conditions on the sample edges are usually considered: the conductive and the convective conditions [5]. 
The conductive boundary conditions between two media denoted " 1 " and " 2 " (conductivity and temperature subscripts in Eqs. (4a, b)) are based on the heat flow conservation and on the temperature continuity. When denoting $\mathbf{n}$ the unit vector perpendicular to the boundary surface at $\mathbf{r}$, these conditions may be written

$$
\begin{gathered}
-\lambda_{1} \mathbf{n} \cdot \nabla T_{1}(t, \mathbf{r})=-\lambda_{2} \mathbf{n} \cdot \nabla T_{2}(t, \mathbf{r}) \\
T_{1}(t, \mathbf{r})=T_{2}(t, \mathbf{r}) .
\end{gathered}
$$

The convective boundary condition contains the coupling constant $h$ which depends on the fluid medium bounding the sample. If we denote $\Phi(t, \mathbf{r})$ the heat flow going from the fluid medium into the sample, this condition is

$$
-\lambda \mathbf{n} \cdot \nabla T(t, \mathbf{r})=h T(t, \mathbf{r})+\Phi(t, \mathbf{r}) .
$$

\subsection{Choice of a Time Analysis}

The two integral transforms used mainly for the time analysis of thermal variations in a solid are the Laplace and the Fourier transforms. The first is particularly suitable when the sample is heated during a short time, as in the so-called "flash method" measurement [6]. The temperature field time Laplace expansion is

$$
T(t, \mathbf{r})=\int_{0}^{\infty} \mathrm{d} s \exp (-s t) T(s, \mathbf{r})
$$

It follows that, according to equation (3), each Laplace transform spectrum coefficient satisfies

$$
\Delta T(s, \mathbf{r})+\frac{s}{a} T(s, \mathbf{r})=-\frac{\sigma(s, \mathbf{r})}{\lambda} .
$$

When the heat source is periodic, it seems preferable to use the time Fourier transform of the temperature, and to write

$$
T(t, \mathbf{r})=\frac{1}{\sqrt{2 \pi}} \int_{-\infty}^{\infty} \mathrm{d} \omega \exp (-i \omega t) T(\omega, \mathbf{r})
$$

Note that although the heat diffusion equation (Eq. (1)) is not a wave propagation equation, we choose here a phase convention which is usual in quantum mechanics and optics for the time Fourier transform [13].

Taking into account equation (3), we may write, concerning the Fourier spectrum coefficients of $T$ and $\sigma$

$$
\Delta T(\omega, \mathbf{r})+\frac{i \omega}{a} T(\omega, \mathbf{r})=-\frac{\sigma(\omega, \mathbf{r})}{\lambda}
$$

In order to simplify and to standardize notations, let us write the spectral coefficients in a uniform way $T(\mathbf{r})$ and $\sigma(\mathbf{r})$. Thus, the temperature Laplace mode becomes $\exp (-s t) T(\mathbf{r})$, while its Fourier mode is denoted $\exp (-i \omega t) T(\mathbf{r})$. With this notation, equations $(7,9)$ both become

$$
\Delta T(\mathbf{r})+K^{2} T(\mathbf{r})=-\frac{\sigma(\mathbf{r})}{\lambda}
$$

where $K$ is a complex number defined according to the chosen time transform

$$
\begin{aligned}
& K=\sqrt{\frac{s}{a}} \text { for the Laplace transform } \\
& K=\sqrt{\frac{i \omega}{a}} \text { for the Fourier transform. }
\end{aligned}
$$


In the case where there is no heat source, equation (10) becomes

$$
\Delta T(\mathbf{r})+K^{2} T(\mathbf{r})=0
$$

in which we recognize a free space wave equation, where $K^{2}$ is square of the (complex) wave vector (not to be confused with the square of the norm).

Thus, we shall first develop from equation (13) a general spectrum of plane waves, and then we shall see the consequences, for that spatial spectrum, of the time-transform choice.

\subsection{General Plane Wave Expansion}

The plane wave expansion [14], used often in near-field optics, can be applied to solve all differential equations with the appearance of the Helmoltz equation, in quantum mechanical, acoustic [15] or thermal areas $[7,16]$. Using Cartesian coordinates, we suppose that the solid sample has no edges in the $x$ and $y$ directions. Under these conditions, we may formulate for each time mode $T(\mathbf{r})=T(x, y, z)$, in a $z=$ constant plane, the $2 \mathrm{D}$ Fourier transform depending on $x$ and $y$. In this way, the time mode may be written as

$$
T(x, y, z)=\frac{1}{2 \pi} \int_{-\infty}^{\infty} \mathrm{d} k_{x} \int_{-\infty}^{\infty} \mathrm{d} k_{y} \tilde{T}\left(k_{x}, k_{y}, z\right) \exp \left[i\left(x k_{x}+y k_{y}\right)\right] .
$$

Each space mode $\tilde{T}\left(k_{x}, k_{y}, z\right)$ must satisfy equation (13). This condition leads to

$$
\frac{\partial^{2} \tilde{T}\left(k_{x}, k_{y}, z\right)}{\partial z^{2}}+\left(K^{2}-k_{x}^{2}-k_{y}^{2}\right) \tilde{T}\left(k_{x}, k_{y}, z\right)=0 .
$$

By using the Laplace or Fourier time transform and then the 2D space Fourier transform, we have changed from a $4 \mathrm{D}$ to a $1 \mathrm{D}$ differential equation. The most general solution of this very classical problem is the double plane wave

$$
\tilde{T}\left(k_{x}, k_{y}, z\right)=\tilde{T}^{+}\left(k_{x}, k_{y}, 0\right) \exp (i \chi z)+\tilde{T}^{-}\left(k_{x}, k_{y}, 0\right) \exp (-i \chi z)
$$

where $\chi$ is the wave vector $z$-component defined as

$$
\chi=\sqrt{K^{2}-k_{x}^{2}-k_{y}^{2}} \text { with } \operatorname{Im}(\chi) \geq 0 .
$$

In the case where the sample can be considered as a half space extending in the $z \geq 0$ range and without thermal source, the second term in the right part of equation (16) disappears and each time-mode can be expanded as a simple spectrum of plane waves [17]

$$
\tilde{T}\left(k_{x}, k_{y}, z\right)=\tilde{T}\left(k_{x}, k_{y}, 0\right) \exp (i \chi z) .
$$

Let us examine the wave vector, the components of which are $k_{x}, k_{y}$ and $\chi$. Its $x$ and $y$ components are always real, because they are the $x$ and $y$ conjugate variables in the 2D spatial Fourier transform. If the time-transform is the Laplace one, $K^{2}$, equal to $s / a$, is real. Thus, the $z$ component $\chi$ can be real or purely imaginary, depending on $k_{x}^{2}+k_{y}^{2}$, as in dielectric media optics. In this case, again as in dielectric optics, the space spectrum $\tilde{T}\left(k_{x}, k_{y}, 0\right)$ is composed of a progressive part and of an evanescent part. Nevertheless, let us remember that each wave, even spatially progressive, is time-damped.

On the other hand, if the time-transform is the Fourier one, $K^{2}$, equal to $i \omega / a$, is complex. It follows that $\chi$ is complex whatever the value of $k_{x}^{2}+k_{y}^{2}$. Thus, in this case, all the waves are damped, in a way which is described below in a detailed manner, and which recalls metal optics. 


\subsection{Reflection and Transmission Coefficients on a Plane Interface}

Equation (16) calls to mind the couple - incident and reflected wave - near a plane interface, in optics or in acoustics. This similarity may be clarified as follows. Let us assume that the sample is semi-infinite, with a plane edge surface at $z=0$. In spite of the big physical differences between the thermal field and the acoustic or the electromagnetic one, by taking into account the boundary conditions (Eqs. (4) or (5)) and the plane waves expansion equation (16), we can define reflection (conductive and convective conditions) and transmission (conductive conditions) coefficients.

Let us consider the conductive conditions equations (4), convenient in the case where the medium extending in the $z \leq 0$ half-space is solid. The problem is to link the temperature field mode propagating in $z \leq 0$ to the one which propagates in $z \geq 0$. To be clear, we note with a " 1 " subscript the physical quantities (wave vector, thermal conductivity, temperature) related to the $z \leq 0$ medium, and with a "2" subscript those related to the $z \geq 0$ medium. Let us write the boundary conditions in the Fourier space, i.e. for the single or double wave related with a given couple $\left(k_{x}, k_{x}\right)$. As in optics or in acoustics, we have in the first medium an incident wave and a reflected one (double wave equation (16)). On the contrary, in the second medium, which is supposed semi-infinite, we have only a transmitted wave (simple wave equation (18)). Thus, the temperature continuity at $z=0$ leads to

$$
\tilde{T}_{1}^{+}\left(k_{x}, k_{y}, 0\right)+\tilde{T}_{1}^{-}\left(k_{x}, k_{y}, 0\right)=\tilde{T}_{2}\left(k_{x}, k_{y}, 0\right) .
$$

Note: for a given time conjugate variable $s$ or $\omega, K_{1}$ and $K_{2}$ are defined following equations (11) or (12), with $a_{1}$ and $a_{2}$ respectively.

In the Fourier space, the heat flow conservation condition (Eq. (4a)) becomes

$$
\lambda_{1} \chi_{1}\left[\tilde{T}_{1}^{+}\left(k_{x}, k_{y}, 0\right)-\tilde{T}_{1}^{-}\left(k_{x}, k_{y}, 0\right)\right]=\lambda_{2} \chi_{2} \tilde{T}_{2}\left(k_{x}, k_{y}, 0\right) .
$$

By combination of equations (19) and (20), we obtain the reflected mode $\tilde{T}_{1}^{-}\left(k_{x}, k_{y}, 0\right)$ and the transmitted mode $\tilde{T}_{2}\left(k_{x}, k_{y}, 0\right)$ depending on the incident one $\tilde{T}_{1}^{+}\left(k_{x}, k_{y}, 0\right)$

$$
\tilde{T}_{1}^{-}\left(k_{x}, k_{y}, 0\right)=\frac{\lambda_{1} \chi_{1}-\lambda_{2} \chi_{2}}{\lambda_{1} \chi_{1}+\lambda_{2} \chi_{2}} \tilde{T}_{1}^{+}\left(k_{x}, k_{y}, 0\right)
$$

and

$$
\tilde{T}_{2}^{+}\left(k_{x}, k_{y}, 0\right)=\frac{2 \lambda_{1} \chi_{1}}{\lambda_{1} \chi_{1}+\lambda_{2} \chi_{2}} \tilde{T}_{1}^{+}\left(k_{x}, k_{y}, 0\right) .
$$

The formal analogy with optics (Fresnel formulas for the T.E. wave) is strong. Thus, it seems convenient to denote "reflection coefficient" the factor multiplying $\tilde{T}_{1}^{+}\left(k_{x}, k_{y}, 0\right)$ in equation (21), and "transmission coefficient" the factor in equation (22).

\subsection{Huygens-Fresnel Principle for the Thermal Waves}

The plane wave spectrum has been linked years ago with the Rayleigh formula expressing the Huygens-Fresnel principle [17]. This appears valid in the case of the thermal diffusion waves just as it is in optics or in acoustics. For temperature field calculations, it could constitute an alternative to the use of equation (18), in the case of a strongly localized field for which the width of the plane wave spectrum creates numerical problems.

Suppose that we know the temperature field on the plane $z=0$. Let us write in this plane the 2D Fourier transform

$$
\tilde{T}\left(k_{x}, k_{y}, 0\right)=\frac{1}{2 \pi} \int_{-\infty}^{\infty} \mathrm{d} x^{\prime} \int_{-\infty}^{\infty} \mathrm{d} y^{\prime} T\left(x^{\prime}, y^{\prime}, 0\right) \exp \left[-i\left(x^{\prime} k_{x}+y^{\prime} k_{y}\right)\right]
$$


Taking into account equations $(14,18,23)$, we obtain

$$
T(x, y, z)=\frac{1}{(2 \pi)^{2}} \iiint \int \mathrm{d} k_{x} \mathrm{~d} k_{y} \mathrm{~d} x^{\prime} \mathrm{d} y^{\prime} T\left(x^{\prime}, y^{\prime}, 0\right) \exp \left\{i\left[\left(x-x^{\prime}\right) k_{x}+\left(y-y^{\prime}\right) k_{y}+z \chi\right]\right\}
$$

which may also be written with the so-called field propagator $G$

$$
T(x, y, z)=\frac{1}{(2 \pi)^{2}} \iint \mathrm{d} x^{\prime} \mathrm{d} y^{\prime} T\left(x^{\prime}, y^{\prime}, 0\right) G\left(x-x^{\prime}, y-y^{\prime}, z\right)
$$

where

$$
\begin{aligned}
G\left(x-x^{\prime}, y-y^{\prime}, z\right) & =\frac{1}{(2 \pi)^{2}} \iint \mathrm{d} k_{x} \mathrm{~d} k_{y} \exp \left\{i\left[\left(x-x^{\prime}\right) k_{x}+\left(y-y^{\prime}\right) k_{y}+z \chi\right]\right\} \\
& =\frac{-i}{(2 \pi)^{2}} \frac{\partial}{\partial z} \iint \mathrm{d} k_{x} \mathrm{~d} k_{y} \frac{\exp \left\{i\left[\left(x-x^{\prime}\right) k_{x}+\left(y-y^{\prime}\right) k_{y}+z \chi\right]\right\}}{\chi} .
\end{aligned}
$$

Let us remember the Weyl formula which is available for $K$ complex as well as for $K$ real, provided that the imaginary part of $K$ is positive [18]

$$
\iint \mathrm{d} k_{x} \mathrm{~d} k_{y} \frac{\exp \left\{i\left[\left(x-x^{\prime}\right) k_{x}+\left(y-y^{\prime}\right) k_{y}+z \chi\right]\right\}}{\chi}=-2 i \pi \frac{\exp \left(i K\left|\mathbf{r}-\mathbf{r}^{\prime}\right|\right)}{\left|\mathbf{r}-\mathbf{r}^{\prime}\right|}
$$

with

$$
\left|\mathbf{r}-\mathbf{r}^{\prime}\right|=\sqrt{\left(x-x^{\prime}\right)^{2}+\left(y-y^{\prime}\right)^{2}+z^{2}} .
$$

Since the condition $\operatorname{Im}\left(K^{2}\right) \geq 0$ is always fulfilled for the thermal waves, equations (25-27) give

$$
T(x, y, z)=-\frac{1}{2 \pi} \int_{-\infty}^{\infty} \mathrm{d} x^{\prime} \int_{-\infty}^{\infty} \mathrm{d} y^{\prime} T\left(x^{\prime}, y^{\prime}, 0\right) \frac{\partial}{\partial z}\left[\frac{\exp \left(i K\left|\mathbf{r}-\mathbf{r}^{\prime}\right|\right)}{\left|\mathbf{r}-\mathbf{r}^{\prime}\right|}\right] .
$$

Equation (29) is the Rayleigh formula corresponding to the Huygens-Fresnel principle in the case of a plane boundary condition. We notice its validity for the thermal diffusion problem. The obliquity factor is in the integrand of equation (29). This could be the point of departure for the formal approach of the thermal diffraction by a narrow aperture [19].

\section{The Harmonic Thermal Field}

\subsection{Experimental Interest}

Nowadays, a.c. temperature measurement is a very important tool for non destructive control. The spatial damping of a thermal harmonic wave depends on its frequency. Thus, low and high frequencies do not furnish the same information about the sample. In particular, a variable thickness of the sample material can be made "thermally visible". That is why, in the following part of this paper, we consider exclusively the case of the harmonic temperature field.

\subsection{The Diffusion Length Related to the Time Frequency}

The Fourier time-transform provides a spectrum of damped waves characterized by (see Eq. (11))

$$
K=\sqrt{\frac{i \omega}{a}}=(1+i) \sqrt{\frac{\omega}{2 a}} .
$$


Let us consider a thermal wave with a $z$-directed wave vector $\left(k_{x}=k_{y}=0\right)$, diffusing from plane A to plane B in a semi-infinite solid medium located in $z \geq 0$. According to (17) and (18), the temperature on B may be written

$$
T(\mathrm{~B})=T(\mathrm{~A}) \exp \left[i K\left(z_{\mathrm{B}}-z_{\mathrm{A}}\right)\right]
$$

which gives, from equation (30),

$$
T(\mathrm{~B})=T(\mathrm{~A}) \exp \left[(-1+i) \sqrt{\frac{\omega}{2 a}}\left(z_{\mathrm{B}}-z_{\mathrm{A}}\right)\right] .
$$

In equation (32), the $z_{\mathrm{B}}-z_{\mathrm{A}}$ distance on which the complex temperature phase increases by one radian, while its amplitude is divided by $e$, is called the "diffusion length", and denoted $\mu$

$$
\mu=\sqrt{\frac{2 a}{\omega}} .
$$

Thus, the heat source modulation frequency chosen makes the sample "thermally thick" or "thermally thin". When the heat source modulation has a low frequency, the surface temperature depends on the bulk structure of the sample. On the contrary, with a high modulation frequency, the surface temperature depends only on the surface structure.

This selective sensibility can be used for solving the inverse problem. For example, Lan et al. [1] propose a multilayer method for reconstituting the thermal conductivity profile $\kappa(z)$ of a one-dimensional material. Several surface temperature measurements are made with decreasing frequency heat sources. Each measurement is assumed to provide information about a depth equal to the diffusion length concerned. Thus, each measurement provides a new independent line in a linear system. The conductivity profile is obtained by solving this system.

\subsection{The Harmonic Thermal Wave Vector}

As said above, in a homogeneous isotropic medium without thermal sources, the harmonic thermal wave vector $\mathbf{K}$ following from the $2 \mathrm{D}$ spatial transform satisfies

$$
K^{2}=i \frac{\omega}{a}=k_{x}^{2}+k_{y}^{2}+\chi^{2}
$$

In the case of electromagnetic or mechanical waves, a complex wave vector square is characteristic of an absorptive medium. In optics, where we have $K=n \omega / c$, the refractive index $n$ is complex. But generally, the imaginary part of $n$ is much smaller than its real part. The equality between them is obtained only very near the resonance. On the contrary, in thermal detection, according to equation (30), the real and the imaginary parts of $K$ are always equal.

It follows that all the harmonic temperature plane waves are strongly damped, even those with $k_{x}$ and $k_{y}$ close to zero. Indeed, for $k_{x}=k_{y}=0$, according to equation (32), when the temperature phase increases by $2 \pi$, the amplitude is divided by more than 500 . Thus, although thermal-wave interference fringes do exist [20], they do not appear as often as optical-wave interferences. However they are responsible, for example, for edge effects.

We show in Figures 1a, b, c the real and imaginary parts of $\chi$ depending on $k_{x}$, for $k_{y}=0$. The wave vector pictured in Figure 1a is related to light propagation in a transparent medium (real refractive index) - we see, in the "horizontal" plane, the half-circle followed by the extremity of the real wave vector related to low spatial frequencies (progressive field) and, in the "vertical" plane, the two hyperbolic branches where the extremity of the complex wave vector related to the high spatial frequencies (evanescent field) is situated. Figure 1b shows 
a)

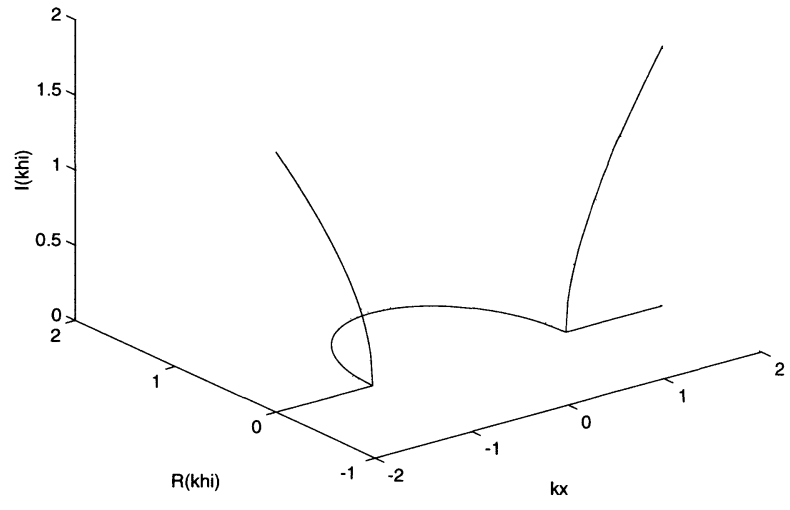

b)

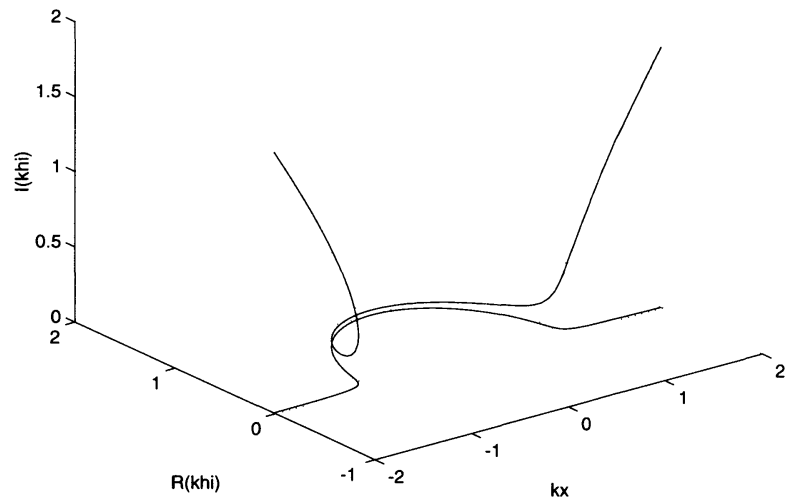

c)

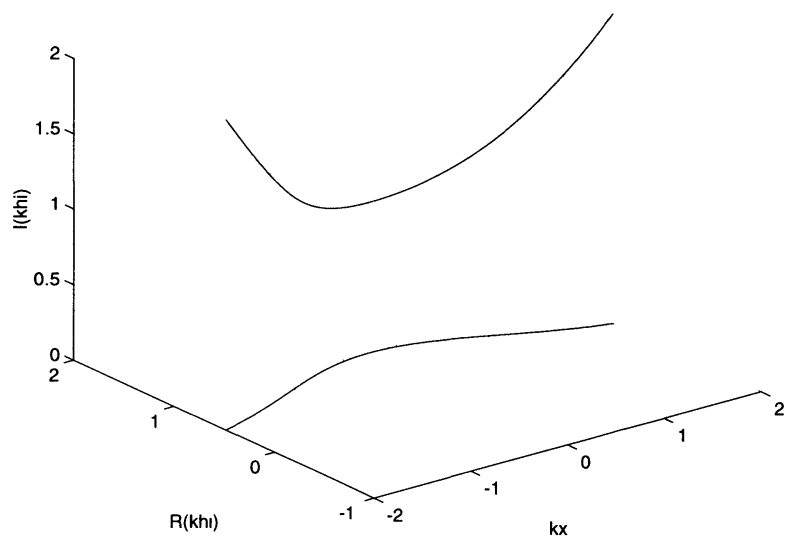

Fig. 1. - The extremity of the complex wave-vector in the case where $k_{y}=0$, for dielectric optics (a), metal optics (b), and thermal harmonic field (c). In (b), the refractive index is $n=1.5+0.05 i$. In order to make comparisons, we show dimensionless components $k_{x} / R(K), R(\chi) / R(K)$ and $I(\chi) / R(K)$. Dashed lines show the wave-vector projection in the $z=0$ plane. 
an example of an optical wave vector in the case of an absorptive medium (complex refractive index), and Figure 1c shows an example of a thermal wave vector. Whatever the $x$-component, the wave vector $\mathbf{K}$ has a complex $z$-component the imaginary part of which increases with the absolute value of $k_{x}$. For $k_{x}$ increasing to the infinity, the extremity of $\mathbf{K}$ describes an hyperbola, as in the optical case.

\section{Temperature Field Generated by Particular Sources}

\subsection{Thermal Problem Specificities}

The thermal problem is often more simple than the optical one, not only because the field is scalar, but also because of the way in which thermal sources are generated. For example, when using a laser beam to heat the sample, an optical wavelength for which the sample is very absorptive is chosen. Thus, it is often possible to consider that the conversion of optical into thermal energy occurs at the surface: the heat source is purely superficial. For the calculation, such a heat source does not appear in right part in equation (3) but as a heat flow in the boundary condition equation (5), which is simpler. Section 5 shows a real example of that situation, where, despite the presence of a heat source in the experiment, the mathematical formulation is that of a "without source problem".

Concerning the bulk heat source, it may be obtained in a homogeneous material, for example by the Joule effect, or by progressive absorption of a laser beam. In such a case, the alternative temperature field variations are obtained from equation (9).

\subsection{The Flat Internal Source}

When it is not on the sample edge, the plane heat source can no longer be considered as a boundary condition. It appears as a term on the right-hand side of equation (9). Calculations related to the plane heat source are of a great theoretical interest, particularly because the most general 3D source may be modeled as a plane elementary source integral. We examine here a plane internal harmonic source heating a homogeneous isotropic medium, without edges in the $x$ and $y$ directions. For such a source located in the $z=z_{\mathrm{S}}$ plane, the harmonic diffusion equation (9) becomes

$$
\Delta T(\mathbf{r})+\frac{i \omega}{a} T(\mathbf{r})=-\frac{\sigma(x, y)}{\lambda} \delta\left(z-z_{\mathrm{S}}\right)=-G(\mathbf{r}) .
$$

Let us take the 3D spatial Fourier transform of the temperature and heat source fields, defined as follows

$$
\tilde{\tilde{T}}(\mathbf{k})=\frac{1}{(2 \pi)^{3 / 2}} \iiint \mathrm{d} \mathbf{r} \exp [-i \mathbf{k r}] T(\mathbf{r}) .
$$

In the Fourier space, equation (35) becomes

$$
-k^{2} \tilde{\tilde{T}}(\mathbf{k})+\frac{i \omega}{a} \tilde{\tilde{T}}(\mathbf{k})=-\tilde{\tilde{G}}(\mathbf{k})
$$

So, the inverse Fourier transform provides the temperature field

$$
T(\mathbf{r})=\frac{1}{(2 \pi)^{3 / 2}} \iiint \mathrm{d} \mathbf{k} \exp [i \mathbf{k r}] \frac{\tilde{G}(\mathbf{k})}{k^{2}-i \omega / a} .
$$

It is worth noting the real values of the three components of the reciprocal vector $\mathbf{k}$. This purely real vector does not coincide with the complex thermal wave vectors which appear in the $1 \mathrm{D}$ 
problem equation (31) or in the harmonic plane waves spectrum described in the preceding section. Equation (38) is very general. It is valid whatever the shape of the heat source. As we consider here the flat source case, the $\delta$ Dirac-function properties lead to the 3D Fourier transform

$$
\tilde{\tilde{G}}(\mathbf{k})=\tilde{\sigma}\left(k_{x}, k_{y}\right) \frac{\exp \left(-i k_{z} z_{\mathrm{S}}\right)}{\sqrt{2 \pi}} .
$$

Equation (39) is introduced in equation (38) to obtain

$$
T(\mathbf{r})=\frac{1}{2 \pi} \iint \mathrm{d} k_{x} \mathrm{~d} k_{y} \exp \left[i\left(x k_{x}+y k_{y}\right)\right] \tilde{\sigma}\left(k_{x}, k_{y}\right) I\left(k_{x}, k_{y}, z\right)
$$

where

$$
I\left(k_{x}, k_{y}, z\right)=\frac{1}{2 \pi} \int \mathrm{d} k_{z} \frac{\exp \left[i k_{z}\left(z-z_{\mathrm{S}}\right)\right]}{k_{z}^{2}-\chi^{2}} \text { with } \chi=\sqrt{\frac{i \omega}{a}-k_{x}^{2}-k_{y}^{2}} .
$$

The integration of $I$ can be analytically performed (see Appendix). It leads to

$$
I\left(k_{x}, k_{y}, z\right)=\frac{i \pi}{\chi} \exp \left[i \chi\left|z-z_{\mathrm{S}}\right|\right]
$$

Thus, according to equation (42), the plane wave spectrum of the temperature field generated in the sample can be written

$$
T(\mathbf{r})=\frac{i}{4 \pi \kappa} \iint \mathrm{d} k_{x} \mathrm{~d} k_{y} \tilde{\sigma}\left(k_{x}, k_{y}\right) \frac{\exp \left[i\left(x k_{x}+y k_{y}+\left|z-z_{\mathrm{S}}\right| \chi\right)\right]}{\chi} .
$$

For a given plane source, equation (43) is directly usable by a FFT algorithm to calculate $T(\mathbf{r})$ at any point in an unbounded medium.

The point source constitutes an interesting special case. A source term

$$
G(\mathbf{r})=\frac{p}{\lambda} \delta\left(x-x_{\mathrm{S}}\right) \delta\left(y-y_{\mathrm{S}}\right) \delta\left(z-z_{\mathrm{S}}\right)
$$

leads to

$$
T(\mathbf{r})=\frac{i p}{8 \pi^{2} \lambda} \iint \mathrm{d} k_{x} \mathrm{~d} k_{y} \frac{\exp \left[i\left(x k_{x}+y k_{y}+\left|z-z_{\mathrm{S}}\right| \chi\right)\right]}{\chi} .
$$

Here, the 2D inverse Fourier transform may be analytically performed with the help of the Weyl's theorem. We obtain the well-known Green's function for equation (35)

$$
T(\mathbf{r})=\frac{p}{4 \pi \lambda} \frac{\exp (i K R)}{R}
$$

with

$$
K=\sqrt{\frac{i \omega}{a}} \text { and } R=\sqrt{x^{2}+y^{2}+\left(z-z_{\mathrm{S}}\right)^{2}} .
$$

Comparison between equations (45) and (46) sheds light on the characteristics of the plane waves spectrum, where the decrease of the temperature field is entirely described by the propagator in the $z$ direction. This is a common feature of near field or metal optics. 


\subsection{Exponentially Decreasing 3D Thermal Source}

The temperature field generated by a general harmonic 3D heat source is formally given by equation (38). The numerical implementation of equation (38) is laborious in practice, because of the $3 \mathrm{D}$ Fourier transform. Fortunately, the 3D sources appearing in photothermal imaging are generally of a particular kind for which another approach is possible, i.e. they are exponentially decreasing, according to the Beer's law [21]. Let us consider a solid sample extending in the half-space $z \geq 0$ and heated by a modulated laser beam propagating from the $z<0$ free half-space. At any depth in the sample, the thermal source, proportional to the light intensity, is exponentially decreasing. Thus, from equation (9), the thermal diffusion equation is

$$
\Delta T(x, y, z)+\frac{i \omega}{a} T(x, y, z)=-\frac{\sigma(x, y)}{\lambda} \exp (i \eta z)
$$

where $\eta$ is a complex number with positive real and imaginary parts. On the $z=0$ edge, the temperature field satisfies the convective boundary condition equation (5). By taking the 2D Fourier transform of equation (48), we obtain the equation which links each harmonic temperature mode $\tilde{T}\left(k_{x}, k_{y}, z\right)$ to the corresponding source mode

$$
\frac{\partial^{2} \tilde{T}\left(k_{x}, k_{y}, z\right)}{\partial z^{2}}+\left(\frac{i \omega}{a}-k_{x}^{2}-k_{y}^{2}\right) \tilde{T}\left(k_{x}, k_{y}, z\right)=-\frac{\tilde{\sigma}\left(k_{x}, k_{y}\right)}{\lambda} \exp (i \eta z) .
$$

A well-known particular solution of equation (49) can be formulated, depending on $\chi$

$$
\tilde{T}_{\mathrm{S}}\left(k_{x}, k_{y}, z\right)=-\frac{\tilde{\sigma}\left(k_{x}, k_{y}\right)}{\lambda\left(\chi^{2}-\eta^{2}\right)} \exp (i \eta z) .
$$

The general solution of equation (49) is the sum of equation (50) and of a free-space wave like the one formulated in equation (18). This free-space wave has to be chosen in such a way that the general solution satisfies the convective boundary condition equation (5) written in the Fourier space. Depending on the unknown spectrum coefficient $\tilde{T}_{0}$, the equation to be satisfied is

$$
-i \lambda \chi \tilde{T}_{0}\left(k_{x}, k_{y}, 0\right)+\frac{i \eta \tilde{\sigma}\left(k_{x}, k_{y}\right)}{\lambda\left(\chi^{2}-\eta^{2}\right)}=h\left[\tilde{T}_{0}\left(k_{x}, k_{y}, 0\right)-\frac{\tilde{\sigma}\left(k_{x}, k_{y}\right)}{\lambda\left(\chi^{2}-\eta^{2}\right)}\right] .
$$

From equation (51), it is easy to deduce the convenient free-space wave

$$
\tilde{T}_{0}\left(k_{x}, k_{y}, 0\right)=\frac{h+i \eta \lambda}{h+i \chi \lambda} \frac{\tilde{\sigma}\left(k_{x}, k_{y}\right)}{\lambda\left(\chi^{2}-\eta^{2}\right)} .
$$

From equations $(50,52)$ we then deduce the total plane wave spectrum of the temperature field generated in the sample, i.e. at $z \geq 0$

$$
\tilde{T}\left(k_{x}, k_{y}, z\right)=\frac{\tilde{\sigma}\left(k_{x}, k_{y}\right)}{\lambda\left(\chi^{2}-\eta^{2}\right)}\left[\frac{h+i \eta \lambda}{h+i \chi \lambda} \exp (i \chi z)-\exp (i \eta z)\right] .
$$

It is sufficient to perform the inverse 2D Fourier transform of equation (53) to obtain the temperature map in the sample.

\section{Thermal Wave Propagation in a Multilayered Sample: Comparison Between Experimental and Theoretical Results}

Within the framework of general thermal studies, we are interested in photothermal effects at the nanoscale. Our approach is to collect the complete thermal signature of the sample under 


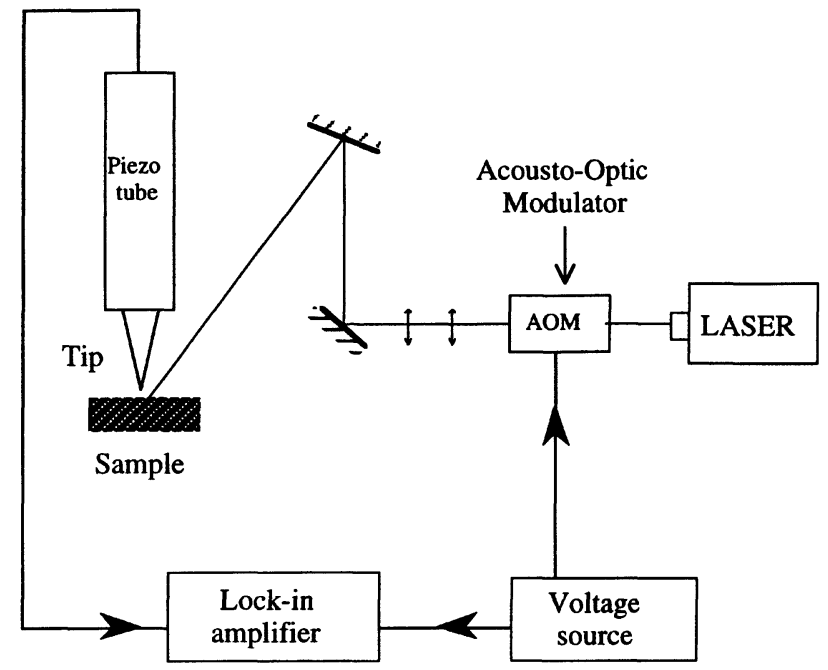

Fig. 2. - Schematic of the experimental setup for the investigation of thermal effects.

laser irradiation. We use the Scanning Tunnelling Microscope (STM) as a non-destructive test to investigate thermal properties of materials [22]. With a view to obtain the thermal signature, experimental results are compared with theoretical results. In our analytical method, the temperature distribution is obtained by solving the heat diffusion harmonic equation (7). The treatment explains the propagation of thermal waves in multilayered structures. Next, a one-dimensional treatment to obtain the thermal expansion of the sample by integrating the temperature distribution is used.

\subsection{Experimental Configuration}

The experimental configuration is shown in Figure 2. The light intensity of an $\mathrm{Ar}^{+}$laser at $488 \mathrm{~nm}$ is periodically modulated by means of an acousto-optic cell. Focused on the sample by a lens, the beam reaches a spot diameter of $50 \mu \mathrm{m}$ and is shifted over the sample using a system of mirrors. Our experiment operates in ambient air and the tip is held at a fixed location. When the sample surface is irradiated, periodic heating causes periodic thermal expansion of the surface at the modulation frequency. The STM is operated in the constant-current mode. Thus, the feedback loop which controls the tip position keeps the tip-sample distance constant. Consequently, the piezoelectric tube voltage $V_{z}$ follows the periodic motion of the surface so as to maintain constant this tip-sample distance. In order to measure the a.c. component of sample surface deformation, the piezo voltage $V_{z}$ is detected by a lock-in technique. In this way, the amplitude and the phase lag of the photothermal displacement relative to the illumination are obtained.

The sample used is a thin gold film $(180 \mathrm{~nm})$ evaporated on a copper substrate. The holder is in PVC to avoid heat losses. Figure 3 shows the thermal signature of the sample when the power of the laser light is $10 \mathrm{~mW}$ and the frequency modulation is fixed at $70 \mathrm{~Hz}$. Figure 3 presents the phase lag component versus the distance between the laser spot and the tip STM. 


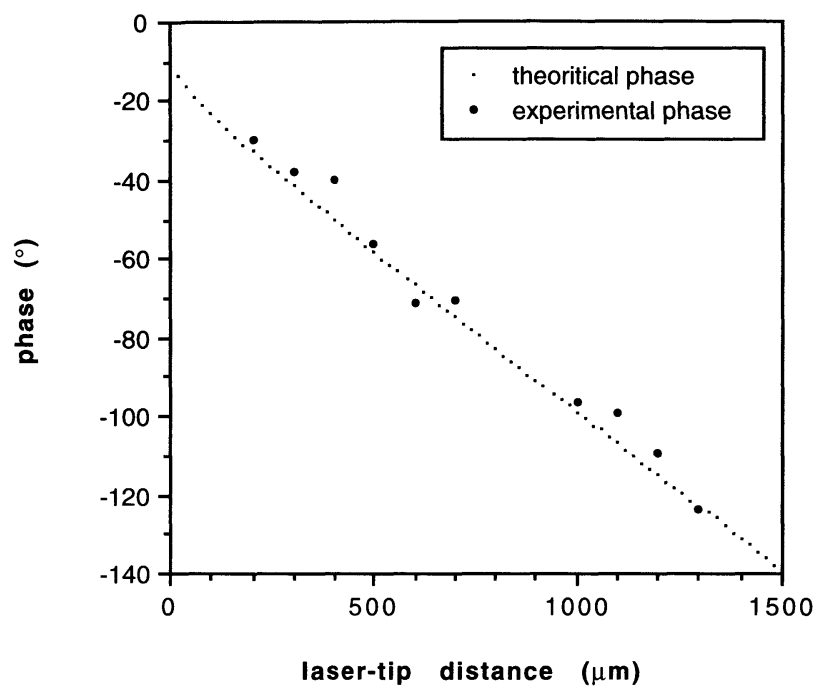

Fig. 3. - Comparison of experimental and theoretical phase of the thermal expansion a.c. component of the sample.

\subsection{Analytical Method}

The sample is assumed to be a bilayered medium with semi-infinite copper layer. The superficial layer composed of gold is opaque and the laser beam is absorbed at the surface sample. To take into account the surface absorption, the source term must be placed into the boundary condition at the surface sample. Thus, the heat diffusion equation for the periodic component of the temperature (Eq. (9)) becomes

$$
\Delta T(\mathbf{r}, z)+\frac{i \omega}{a} T(\mathbf{r}, z)=0
$$

with the convention of an $\mathrm{e}^{-i \omega t}$ time dependence: $T(t, \mathbf{r})=T(\mathbf{r}) \mathrm{e}^{-i \omega t} \cdot \mathbf{r}$ is the position coordinate: $\mathbf{r}(x, y)$ and is now independent of $z$.

To solve the heat diffusion equation, the boundary conditions to be satisfied are:

i) at $z=0$, on the sample surface: the condition of Neumann is applied (Eq. (5)) with the coefficient $h$ neglected

$$
-\lambda_{1} \frac{\partial T(\mathbf{r}, z=0)}{\partial z}=S(\mathbf{r})
$$

in which $S(\mathbf{r})$ is the source term with the Gaussian spatial distribution provided by the laser beam (TEM00):

$$
S(\mathbf{r})=\frac{P_{\mathrm{abs}}}{\pi r_{0}^{2}} \exp \left(-\frac{2 \mathbf{r}^{2}}{r_{0}^{2}}\right)
$$

$P_{\text {abs }}$ is the absorbed power light, and $r_{0}$ the laser spot radius at $\left(1 / e^{2}\right)$.

ii) The interface conditions must be considered at $z=L$, where $L$ is the thickness of the gold layer: the continuity of temperature and the conservation of flux

$$
T_{1}(\mathbf{r}, L)=T_{2}(\mathbf{r}, L) \text { and }-\lambda_{1}\left(\frac{\partial T_{1}(\mathbf{r}, z)}{\partial z}\right)=-\lambda_{2}\left(\frac{\partial T_{2}(\mathbf{r}, z)}{\partial z}\right) .
$$


Indexes 1 and 2 correspond to the respective medium, gold thin film and copper.

iii) at $z=\infty, T_{2}(\mathbf{r}, z)=0$.

We applied the Fourier transform of the heat diffusion equation and the boundary conditions (Eqs. $(54,56)$ ). Equation (54) for each medium becomes:

$$
\frac{\partial^{2} \tilde{T}\left(k_{x}, k_{y}, z\right)}{\partial z^{2}}+\chi^{2} \tilde{T}\left(k_{x}, k_{y}, z\right)=0
$$

in which $k_{x}, k_{y}$ are variables of Fourier space and $\chi$ is defined by equation (17). Equation (58) has a solution of the form:

$$
\begin{aligned}
& \tilde{T}_{1}\left(k_{x}, k_{y}, z\right)=C_{\mathrm{i}}\left(k_{x}, k_{y}\right) \exp \left(i \chi_{1} z\right)+C_{\mathrm{r}}\left(k_{x}, k_{y}\right) \exp \left(-i \chi_{1} z\right) \\
& \tilde{T}_{2}\left(k_{x}, k_{y}, z\right)=C_{\mathrm{t}}\left(k_{x}, k_{y}\right) \exp \left(i \chi_{2} z\right) .
\end{aligned}
$$

$\tilde{T}_{1}$ and $\tilde{T}_{2}$ are the respective temperature components of the space Fourier transform in the bilayered sample. The problem is treated as wave propagation. Consequently, we introduce $C_{\mathrm{i}}$, $C_{\mathrm{r}}$ and $C_{\mathrm{t}}$ terms for the incident, reflected and transmitted waves in Fourier space. The Fourier transform of the boundary conditions (Eqs. $(55,57)$ ) allows us to determine expressions for the $C_{\mathrm{i}}, C_{\mathrm{r}}$ and $C_{\mathrm{t}}$ terms:

$$
C_{\mathrm{i}}\left(k_{x}, k_{y}\right)=i \frac{P_{\mathrm{abs}}}{2 \lambda_{1}} \frac{1}{(1-\mathcal{R})} \exp \left[-\frac{r_{0}^{2}}{8}\left(k_{x}^{2}+k_{y}^{2}\right)\right]
$$

and

$$
\begin{aligned}
& C_{\mathrm{r}}\left(k_{x}, k_{y}\right)=\mathcal{R} C_{\mathrm{i}}\left(k_{x}, k_{y}\right) \\
& C_{\mathrm{t}}\left(k_{x}, k_{y}\right)=\mathcal{T} C_{\mathrm{i}}\left(k_{x}, k_{y}\right)
\end{aligned}
$$

with $\mathcal{R}$ the reflection coefficient and $\mathcal{T}$ the transmission coefficient

$$
\mathcal{R}=\frac{\lambda_{1} \chi_{1}-\lambda_{2} \chi_{2}}{\lambda_{1} \chi_{1}+\lambda_{2} \chi_{2}} \exp \left[2 i \chi_{1} L\right] \quad \mathcal{T}=\frac{2 \lambda_{1} \chi_{1}}{\lambda_{1} \chi_{1}+\lambda_{2} \chi_{2}} \exp \left[i\left(\chi_{1}-\chi_{2}\right) L\right]
$$

Finally, the temperature distribution in the Fourier space is given by

$$
\begin{gathered}
\tilde{T}_{1}\left(k_{x}, k_{y}, z\right)=i \frac{P_{\mathrm{abs}}}{2 \pi \lambda_{1}} \frac{1}{\chi_{1}(1-\mathcal{R})} \exp \left[-\frac{r_{0}^{2}}{8}\left(k_{x}^{2}+k_{y}^{2}\right)\right]\left\{\exp \left[i \chi_{1} z\right]+\mathcal{R} \exp \left[-i \chi_{1} z\right]\right\} \\
\tilde{T}_{2}\left(k_{x}, k_{y}, z\right)=i \frac{P_{\mathrm{abs}}}{2 \pi \lambda_{1}} \frac{\mathcal{T}}{\chi_{1}(1-\mathcal{R})} \exp \left[-\frac{r_{0}^{2}}{8}\left(k_{x}^{2}+k_{y}^{2}\right)\right] \exp \left[i \chi_{2} z\right] .
\end{gathered}
$$

To obtain temperature distribution in the real space, the inverse Fourier transform must be applied to equations $(62 \mathrm{a}, \mathrm{b})$. We are, however, interested in comparing the sample thermal expansion with the experimental value. To determinate this, we introduce the usual expression for the thermal expansion for each medium:

$$
\Delta Z_{1}=\beta_{1} \int_{0}^{L} T_{1}(\mathbf{r}, z) \mathrm{d} z \quad \Delta Z_{2}=\beta_{2} \int_{L}^{\infty} T_{2}(\mathbf{r}, z) \mathrm{d} z
$$

$\beta_{1}$ and $\beta_{2}$ are thermal expansion coefficients of gold and copper. The resulting sample expansion is the sum of the expansions of each medium, $\Delta Z=\Delta Z_{1}+\Delta Z_{2}$. Although equations (62) give the temperature field, it corresponds to the case of a strongly localized field for which the large width of the plane wave spectrum creates numerical problems (see above, 2.6). Therefore, 
we first integrate over $z$ and then integrate in the Fourier space. We also introduce the Bessel function and obtain the following expressions which have to be evaluated numerically:

$$
\begin{gathered}
\Delta Z_{1}(\mathbf{r})=\beta_{1} \frac{P_{\mathrm{abs}}}{2 \pi \lambda_{1}} \int_{0}^{\infty} \frac{\exp \left(-\frac{r_{0}^{2}}{8} q^{2}\right)}{\chi_{1}^{2}}\left[\frac{\left[\exp \left(i \chi_{1} L\right)-\mathcal{R} \exp \left(-i \chi_{1} L\right)\right]}{(1-\mathcal{R})}-1\right] J_{0}(q \mathbf{r}) q \mathrm{~d} q \\
\Delta Z_{2}(\mathbf{r})=\beta_{2} \frac{P_{\mathrm{abs}}}{2 \pi \lambda_{2}} \int_{0}^{\infty} \frac{\exp \left(-\frac{r_{0}^{2}}{8} q^{2}\right)}{\chi_{1} \chi_{2}}\left[-\frac{\left[\mathcal{T} \exp \left(i \chi_{2} L\right)\right]}{(1-\mathcal{R})}\right] J_{0}(q \mathbf{r}) q \mathrm{~d} q .
\end{gathered}
$$

\subsection{Results}

Experimental results corresponding to the measurement of the thermal expansion phase component of the sample are reported in Figure 3 and compared with our theoretical calculation. A good agreement between theory and experiment is obtained. The sample thermal expansion is investigated by analyzing the phase lag of the thermal signal a.c. component collected by STM and the use of the Fourier transform method allows one to calculate the phase.

\section{Conclusion}

In this paper we have presented theoretical and numerical techniques, based on Fourier nearfield optics, to describe photothermal phenomena in isotropic and homogeneous materials. The above examples show that the Fourier transform is a very powerful tool in theoretical conductive thermal studies. The plane wave decomposition is particularly useful in many alternating thermal field calculations, both at the microscopic and at the macroscopic scale. Furthermore, in thermal diffusion problems, it can be used even when the sample contains heat sources. Among the different integral transforms used to study harmonic thermal diffusion [23, 24], the thermal plane wave expansion can often be implemented numerically without difficulty, even on a personal computer, by using the Fast Fourier Transform algorithms. In special cases (wide spectrum) the numerical implementation is facilitated by using Rayleigh's integral or Bessel functions.

The example described in Section 5 shows the plane wave spectrum used in a practical nanothermic problem, where numerical calculations are performed with the help of Bessel functions.

\section{Appendix}

The integral $I\left(k_{x}, k_{y}, z\right)$ (Eq. (41)) can be easily evaluated in the complex plane. $I\left(k_{x}, k_{y}, z\right)$ can be written

$$
I\left(k_{x}, k_{y}, z\right)=\frac{1}{4 \pi \chi} \int \mathrm{d} k_{z} \exp \left[i k_{z}\left(z-z_{\mathrm{S}}\right)\right]\left(\frac{1}{k_{z}-\chi}-\frac{1}{k_{z}+\chi}\right)
$$

where the simple poles appear. The theorem of residues yields straightforwardly

$$
\begin{aligned}
I\left(k_{x}, k_{y}, z\right)=\frac{i}{2 \chi} \exp \left[i \chi\left(z-z_{\mathrm{S}}\right)\right] & \text { for } z-z_{\mathrm{S}} \geq 0 \\
I\left(k_{x}, k_{y}, z\right) & =\frac{i}{2 \chi} \exp \left[-i \chi\left(z-z_{\mathrm{S}}\right)\right] \text { for } z-z_{\mathrm{S}} \leq 0 .
\end{aligned}
$$




\section{References}

[1] Lan T.T.N., Seidel U. and Walther H.G., J. Appl. Phys. 77 (1995) 4739-4744.

[2] Seidel U., Haupt K., Walther H.G., Burt J.A. and Munidasa M., J. Appl. Phys. 78 (1995) 2050-2056.

[3] Hammiche A., Pollock H.M., Song M. and Hourston D.J., Meas. Sci. Technol. 7 (1996) 142-150.

[4] Oesterschulze E., Stopka M., Ackermann L., Scholz W. and Werner S., J. Vac. Sci. Technol. $B 14$ (1996) 1-6.

[5] Carslaw H.S. and Jaeger J.C., Conduction of heat in solids (2nd ed., Oxford, 1959).

[6] Batsale J.C., Bendada A., Maillet D. and Degiovanni A., Distribution of a thermal contact resistance: inversion using experimental Laplace and Fourier Transformations and an asymptotic expansion, Inverse problems in Engineering: Theory and Practice, ASME (1993) pp. 139-146.

[7] Vaez Iravani M. and Wickramasinghe H.K., J. Appl. Phys. 58 (1985) 122-131.

[8] Friedrich K., Haupt K., Seidel U. and Walther H.G., J. Appl. Phys. 72 (1992) 3759-3764.

[9] Oesterschulze E., Stopka M. and Kassing R., Microel. Eng. 24 (1994) 107-112.

[10] Vernotte P., C. R. Hebdo. Séan. Acad. Sci. Paris 247 (1958) 2103-2105.

[11] Joseph D.D. and Preziosi L., Rev. Mod. Phys. 61 (1989) 41-73.

[12] Qiu T.Q., Juhasz T., Suarez C., Bron W.E. and Tien C.L., Int. J. Heat Mass Transfer 37 (1994) 2789-2797.

[13] Mandelis A., J. Opt. Soc. Am. A 6 (1989) 298-308.

[14] Nieto-Vesperinas M., Scattering and Diffraction in Physical Optics (Wiley, New- York, 1993).

[15] Nikoonahad M. and Ash E.A., Acoustical Imaging, E.A. Ash and C.R. Hill, Ed. (Plenum, New-York, 1982) p. 47.

[16] Ozisik N., Boundary Values Problems of Heat Conduction (Dover, New-York, 1989).

[17] Lalor E., J. Opt. Soc. Am. 58 (1968) 1235-1237.

[18] Stratton J.A., Electromagnetic theory (Mc Graw-Hill, New-york, 1941) p. 411.

[19] Mandelis A., J. Opt. Soc. Am. A 6 (1989) 298-308.

[20] Mandelis A. and Leung K.F., J. Opt. Soc. Am. 8 (1991) 186-200.

[21] Pérez J.P., Optique géométrique et ondulatoire (Masson, Paris, 1994) p. 143.

[22] Grafström S., Kowalski J., Neumann R., Probst O. and Wörtge M., J. Vac. Sci. Tecnol. B 9-2 (1991) 568.

[23] Ozisik N., Heat conduction (Wiley, New-York, 1990).

[24] Mandelis A., J. Math. Phys. 26 (1985) 2676-2686. 